\title{
Palliative radiation therapy in bladder cancer: a matter of dose, techniques and patients' selection
}

\author{
Barbara A. Jereczek-Fossa ${ }^{1,2}$, Giulia Marvaso ${ }^{1}$ \\ ${ }^{1}$ Division of Radiotherapy, IEO, European Institute of Oncology IRCCS, Milan, Italy; ${ }^{2}$ Department of Oncology and Hemato-Oncology, University \\ of Milan, Milan, Italy \\ Correspondence to: Barbara A. Jereczek-Fossa. Department of Oncology and Hemato-Oncology, University of Milan, Via Ripamonti 435, Milan, Italy. \\ Email: barbara.jereczek@ieo.it. \\ Provenance: This is an invited article commissioned by the Guest Section Editor Dr. Xiao Li (Department of Urology, Jiangsu Cancer Hospital, \\ Jiangsu Institute of Cancer Research, Nanjing Medical University Affiliated Cancer Hospital, Nanjing, China). \\ Comment on: Ali A, Song YP, Mehta S, et al. Palliative Radiation Therapy in Bladder Cancer-Importance of Patient Selection: A Retrospective \\ Multicenter Study. Int J Radiat Oncol Biol Phys 2019;105:389-93.
}

Submitted Oct 28, 2019. Accepted for publication Nov 05, 2019.

doi: 10.21037/apm.2019.11.02

View this article at: http://dx.doi.org/10.21037/apm.2019.11.02

Palliative care privileges comfort over healing, with the main intent on management of symptoms such as pain or bleeding. Usually palliative cares are reserved for patients with a diagnosis of terminal illness with less than 6-month life expectancy when the curative treatments are no longer possible (1).

Palliative care has been studied in many types of cancer, demonstrating a consistent improvement in quality of life (QoL) (2,3). Its role in management of patients with bladder cancer is still controversial, even if these patients, especially those with locally advanced disease, present persistent symptoms that dramatically affect their QoL.

In a recent published study by Hugar et al. the use of palliative care in muscle invasive bladder cancer (MIBC) resulted to be quite limited in the USA, with less than $4 \%$ of patients receiving any kind of supportive treatment, regardless of solid proof for integrating its utilization into standard oncological care (4). This fact could be explained by the lack of dedicated guidelines and the presence of only general palliative care guidelines from the European Association of Urology (5).

Understanding palliative care use in MIBC patients is becoming crucial within identifying those who might likely benefit the most.

In the $20-30 \%$ of patients who have potentially fatal MIBC, we have to distinguish two categories of patients: those with potentially curable disease but considered too frail to undergo radical treatments (such as radical cystectomy or trimodality treatment), and others with a disease stage too advanced to offer curative treatment. Both populations however, suffer from severe local symptoms from their disease, with hematuria, and dysuria irritative bladder, which possibly require help for the duration of their survival (6).

Palliative pelvic radiotherapy (PRT) plays a role in these symptoms management and in local control of disease (7).

Ali et al. published a study aiming to investigate the efficacy of PRT in patients with bladder cancer and to identify factors associated with treatment outcome (8). The authors pointed out the very important question of selection in order to identify patients who really can have a benefit with PRT.

This retrospective analysis included 241 patients with a diagnosis of MIBC at different stages, treated with conventional 3D conformal radiotherapy (3D CRT). Median age was of 80 years (range, 41-97). A treatment was considered "futile" when a patient died within 30 days since PRT completion or was not able to complete the course of treatment. The median overall survival (mOS), from the end of RT to death by any cause, was 5 months ( $95 \%$ CI: 4.4-6.2), with $20.3 \%$ of patients surviving longer than 12 months ( 1 in 5 patients).

The mOS were 3.7 months (95\% CI: $1.7-6.3$ ) and 3.2 months (95\% CI: $2.5-4.8$ ) for locally advanced (stage 
III) and late stage (stage IV, recurrence), respectively.

Following the multivariate analysis, longer survival were associated with ECOG PS $\leq 2$, earlier stage of disease (stage $\leq \mathrm{II})$ and higher ACE-27 score $(\geq 2)$. ACE-27 score was a 27 -item validated comorbidity index for use with cancer patients (8). So, patient-related factors were assumed to have a greater impact on potential treatment benefit.

An important consideration should be done about the symptoms control, at the end of RT 75\% (150 of 200) of alive patients were symptomatic, and 53\% among them (80 of 150) experienced an improvement after treatment in hematuria, in local symptoms (i.e., urinary frequency and dysuria) and pain score $(54.1 \%, 56.8 \%$ and $47.6 \%$, respectively). Therefore the effect of PRT was not immediate.

The 30-day mortality after PRT was $18 \%$ (44 patients). A further 7\% (17 patients) died before completing planned PRT and another 7\% (16 patients) did not complete PRT as prescribed.

In conclusion, good performance status patients with earlier stage disease and few comorbidities survived longer and PRT was an effective, well-tolerated treatment. Thus, for preventing futile treatment, comprehensive assessment and patient selection are crucial.

For what concerns RT schedule there was a quiet wide range of used doses, from $8 \mathrm{~Gy} / 1 \mathrm{Fr}$ to $30 \mathrm{~Gy} / 10 \mathrm{Fr}$. Hypofractionated schemes such as $21 \mathrm{~Gy} / 3 \mathrm{Fr}$ or $20 \mathrm{~Gy} / 5 \mathrm{Fr}$ were also employed. Interestingly, no correlation between the RT schedule and clinical outcomes was found.

If there is limited literature about the use of PRT and reporting bladder cancer outcomes, even more uncertain appears the optimal schedule of RT in advanced MIBC. All the available data derive from retrospective studies and from one multicenter randomized trial. MRC BA09 is the largest randomized trial for patient with bladder cancer undergoing PRT. It enrolled 500 patients considered unsuitable for curative treatment according to their disease stage or comorbidity, comparing to RT schedules: 35 Gy/10 Fr and $21 \mathrm{~Gy} / 3 \mathrm{Fr}(7)$. First endpoint of the study was to evaluate symptomatic improvement after treatment. Change in Bladder- or bowel-related symptoms, from pre and post RT treatment, showed no evidence of differences between the treatment arms. Moreover, no difference in survival between the two schedules was evidenced.

Different PRT protocols are still being used, despite these results, and the dose choice depends on clinicians' experience and preference.

What seems clear in this highly palliative setting is that hypofractionation is a reasonable option in order to increase patient comfort (reducing the time traveling to the hospital). Moreover, the hemostatic effect of RT is seen after few fractions and often intractable hematuria is the main symptom in these patients. The disadvantage of the most common hypofractionated schedules ( $5 \mathrm{~Gy} \times 4,8 \mathrm{~Gy}$ $\times 4$ or 13 Gy $\times 3$ once daily) is the higher risk of toxicity, considering the complexity of the pelvic region and the sensitivity of the bladder itself (9). Therefore, different alternative schedules were studied, such as hypofractionated radiation schedule with a once weekly treatment. The rationale of this approach is to potentially permit a recovery from the acute toxicity, but the main drawback could be the tumor repopulation resulting in a less effective treatment and a worse local control (10). Indeed, several studies supported to spreading the dose over 5-6 weeks, with good clinical outcome and lower toxicity compared to conventional treatment $(11,12)$. More recent data confirmed that the Overall Treatment Time (OTT) does not impact the treatment efficacy of RT in bladder cancer (13). It has to be noticed that in the majority of these studies the planning target volume (PTV) was obtained by expanding the clinical target volume (CTV), corresponding to the whole bladder, with a margin of 1-2 cm. The technique used was 3D CRT.

The whole bladder is conventionally considered as the target volume due to the difficulties in tumor localization, in the accuracy of treatment delivery, and because bladder cancer is usually multifocal. Despite that, targeting treatment to the tumor might give equivalent local control (14), as showed by brachytherapy data, and retrospective data too suggest that bladder healthy tissue sparing reduced the toxicity risk (15).

The reports on reduced bladder volume receiving highdose radiation regards mostly the use of RT in MIBC for curative intent in a multimodality setting. The data published in the randomized non-inferiority trial of the BC2001 Trial (CRUK/01/004) showed equivalent outcomes with partial- and whole-bladder irradiation and even reported trends toward improved local control and survival with partial-bladder treatment (16).

Since these earlier studies, radiation and imaging techniques have evolved. The introduction of both intensity-modulated radiation therapy (IMRT) and imageguided RT (IGRT), has improved conformity of the radiation dose to the target volume and a reduction of the gastrointestinal and genitourinary toxicity (17).

Stereotactic body radiotherapy (SBRT) is also emerging as an option in treating macroscopic disease in 
oligometastatic urothelial cancer with encouraging results in terms of oncological outcome and with acceptable toxicity $(18,19)$. Indeed, these two unique Italian series using extremely conformal RT modality (SBRT) to limited volume oligorecurrent/oligometastatic MIBC (including local recurrence) showed local control rate at 1 year after SBRT of $70 \%$. Only single acute or late low-grade toxicity events were registered.

Bladder cancer cells showed to have a lower alpha-beta ratio very close normal tissues one, according to in vitro data. The low alpha-beta ratio of bladder cancer could provide a radiobiological explanation for the efficacy of hypofractionation and the use of SBRT (20).

SBRT targeting macroscopic bladder tumor could differ from the conventional palliative treatment of the whole bladder in three critical ways: the treatment course is shortened; the target volume is reduced (partial bladder RT) and the daily dose is typically hypofractionated. The intent of this kind of intervention is to optimize symptom (and tumor!) control, minimizing bowel and urinary toxicities, and especially reduce OTT treatment augmenting patients' compliance to RT.

Clinical decisions for PRT in this particular setting of patients depends on different factors including comorbidities, stage, age and life expectancy, but a conscious patients' selection should be followed by an accurate choice in RT modality. Encouraging data come from other palliative scenarios in oncology. For example, high-dose, single fraction SBRT has been recently showed in the prospective randomized phase II study, to offer higher rates of pain response and better local tumor control in patients treated for painful bone metastases than did conventionally fractionated RT delivered con standard techniques (21).

We do believe that the combination of clinical parameters evaluation, together with the improvements in technologies could really increase the use of PRT and improve QoL in the management of incurable bladder cancer.

\section{Acknowledgments}

None.

\section{Footnote}

Conflicts of Interest: The authors have no conflicts of interest to declare.

Ethical Statement: The authors are accountable for all aspects of the work in ensuring that questions related to the accuracy or integrity of any part of the work are appropriately investigated and resolved.

\section{References}

1. Huffman JL, Waheed A, Harmer B. End of Life. 2019 Sep 11. StatPearls (Internet). Treasure Island (FL): StatPearls Publishing; 2019 Jan. Available online: http://www.ncbi. nlm.nih.gov/books/NBK544276/

2. Roeland EJ, Triplett DP, Matsuno RK, et al. Patterns of palliative care consultation among elderly patients with cancer. J Natl Compr Canc Netw 2016;14:439-45.

3. Hui D, Elsayem A, De La Cruz M, et al. Availability and integration of palliative care at us cancer centers. JAMA 2010;303:1054-61.

4. Hugar LA, Lopa SH, Yabes JG, et al. Palliative care use amongst patients with bladder cancer. BJU Int 2019;123:968-75.

5. Paez Borda A, Charnay-Sonnek F, Fonteyne V, et al. European Association of Urology 2014. Available online: https://uroweb.org/wp-content/uploads/25- PainManagement_LR.pdf

6. Lydon A, Duchesne GM. How should we palliate bladder cancer? Clin Oncol (R Coll Radiol) 1992;4:273-4.

7. Duchesne GM, Bolger JJ, Griffiths GO, et al. A randomized trial of hypofractionated schedules of palliative radiotherapy in the management of bladder carcinoma: results of medical research council trial BA09. Int J Radiat Oncol Biol Phys 2000;47:379-88.

8. Ali A, Song YP, Mehta S, et al. Palliative Radiation Therapy in Bladder Cancer-Importance of Patient Selection: A Retrospective Multicenter Study. Int J Radiat Oncol Biol Phys 2019;105:389-93.

9. Cihoric N, Crowe S, Eychmüller S, et al. Clinically significant bleeding in incurable cancer patients: effectiveness of hemostatic radiotherapy. Radiat Oncol 2012;7:132.

10. Widmark A, Flodgren P, Damber JE, et al. A systematic overview of radiation therapy effects in urinary bladder cancer. Acta Oncol 2003;42:567-81.

11. Rostom AY, Tahir S, Gershuny AR, et al. Once weekly irradiation for carcinoma of the bladder. Int J Radiat Oncol Biol Phys 1996;35:289-92.

12. Scholten AN, Leer JW, Collins CD, et al. Hypofractionated radiotherapy for invasive bladder cancer. Radiother Oncol 1997;43:163-9.

13. Pos FJ, Hart G, Schneider C, et al. Radical radiotherapy 
for invasive bladder cancer: What dose and fractionation schedule to choose? Int J Radiat Oncol Biol Phys 2006;64:1168-73.

14. van der Werf-Messing BH, van Putten WL. Carcinoma of the urinary bladder category T2,3NXM0 treated by 40 Gy external irradiation followed by cesium137 implant at reduced dose (50\%). Int J Radiat Oncol Biol Phys 1989;16:369-71.

15. Emami B, Lyman J, Brown A, et al. Tolerance of normal tissue to therapeutic irradiation. Int J Radiat Oncol Biol Phys 1991;21:109-22.

16. Huddart RA, Hall E, Hussain SA, et al. Randomized noninferiority trial of reduced high-dose volume versus standard volume radiation therapy for muscleinvasive bladder cancer: results of the BC2001 trial (CRUK/01/004). Int J Radiat Oncol Biol Phys 2013;87:261-9.

17. Lutkenhaus LJ, van Os RM, Bel A, et al. Clinical results of conformal versus intensity modulated radiotherapy using a focal simultaneous boost for muscle-invasive bladder cancer in elderly or medically unfit patients. Radiat Oncol

Cite this article as: Jereczek-Fossa BA, Marvaso G. Palliative radiation therapy in bladder cancer: a matter of dose, techniques and patients' selection. Ann Palliat Med 2019;8(5):786-789. doi: 10.21037/apm.2019.11.02
2016;11:45.

18. Augugliaro M, Marvaso G, Ciardo D, et al. Recurrent oligometastatic transitional cell bladder carcinoma: is there room for radiotherapy? Neoplasma 2019;66:160-5.

19. Francolini G, Desideri I, Detti B, et al. Stereotactic radiotherapy in oligoprogressive and oligorecurrent urothelial cancer patients: A retrospective experience. Cancer Treat Res Commun 2019;19:100124.

20. Kang jj, Iwamoto KS, Peek EM, et al. The Low Alpha-Beta Ratio of Bladder Cancer: A Rationale for Hypofractionation. Proceedings of the 96th Annual Meeting of the American Radium Society 2014. Available online: https://www.cancernetwork.com/ars-2014/ s036-low-alpha-beta-ratio-bladder-cancer-rationale hypofractionation

21. Nguyen QN, Chun SG, Chow E, et al. Single-Fraction Stereotactic vs Conventional Multifraction Radiotherapy for Pain Relief in Patients With Predominantly Nonspine Bone Metastases: A Randomized Phase 2 Trial. JAMA Oncol 2019;5:872-8. 